\title{
How long can patients with mild or moderate Alzheimer's dementia maintain both the cognition and the therapy of cholinesterase inhibitors: a national population-based study
}

\author{
Y. Sun ${ }^{a, b}$, M.-S. Lail ${ }^{b}$ C.-J. Lü and R.-C. Chen ${ }^{a, c}$ \\ ${ }^{a}$ Department of Neurology, En Chu Kong Hospital; ${ }^{b}$ Institute of Preventive Medicine College of Public Health, National Taiwan University; \\ and ${ }^{\mathrm{c}}$ Taiwan Alzheimer's Disease Association, Taipei, Taiwan
}

\section{Keywords: \\ Alzheimer's \\ dementia, cholinesterase \\ inhibitor, treatment \\ duration}

Received 14 September 2007

Accepted 5 December 2007

\begin{abstract}
The aims of this study were to evaluate the duration of acetylcholinesterase inhibitors (AChEI) utilization as well as the patients' cognition maintenance. This study was using panel data from the Bureau of National Health Insurance (BNHI) of Taiwan from 2001 to 2004. Patients with mild or moderate AD were prescribed AChEI (donepezil, rivastigmine, or galantamine). By the regulation of BNHI, if the score of Mini-Mental Status Examination worsened by more than two points or clinical dementia rating (CDR) worsened by one or more grades in the follow-up every half year, the AChEI treatment would be terminated. Kaplan-Meier product-limit method was used to estimate duration of drug utilization. Regression model was performed to analyse the factors affecting the discontinuation of AChEI treatment. Our results showed female are more and younger than male in mild to moderate Alzheimer's dementia. The mean duration of use of AChEI was 432 days. Only $9.6 \%$ of patients maintained stable cognition tests results with continued drug refill for more than 3 years. Discontinuation rate in older patients (age $\geq 76$ years) was higher than those in younger age $(P=0.0009)$. The average duration for AChEI therapy is around 14 months. The elderly are at high risk for treatment discontinuation.
\end{abstract}

\section{Introduction}

Alzheimer's dementia (AD) was one of the most challenging diseases which make the patients and the caregivers suffering from the physical, psychosocial and economical burden. The economic impacts increase as the cognition impairment getting severe [1-3]. As more clinical trials showed the modest effects of acetylcholinesterase inhibitors (AChEI) on cognition in patients with mild or moderate $\mathrm{AD}[4,5]$, more physician use such drugs to treat the patients with dementia to slow the disease progression. However, such kind of medicine was rather costly. This may cause huge burden in the National Health Budget. Several studies have set-up models in simulated, long-duration cohort to calculate the cost-effectiveness of AChEI [6-10]. But needs data of the long-term drug compliance and proportion of discontinuation on therapy from clinical trials and even some assumptions when modelling these pharmacoeconomic studies. However, compliance rate during closely monitored studies might be far higher than in

Correspondence: Dr Yu Sun, Department of Neurology, En Chu Kong Hospital, No. 399, Fuhsin Road, San-shia Town, Taipei 23702, Taiwan (tel.: 886226723456 extn 6657; fax: 8862 26730920; e-mail: sunyu.jj.lu@gmail.com). real-world situation. It is important to present the naturalistic data of the duration of maintaining both cognition function and AChEI therapy in patient with AD.

In this study, we obtained the national populationbased and long-term follow-up data of all the patients diagnosed with mild or moderate $\mathrm{AD}$, who had treatment of AChEI and serial check-ups of cognition. Our aims were to assess the proportion of different ages and sex in the patients with mild or moderate AD who start AChEI treatment; to estimate the AChEI usage duration before them entering to severe stage; to assess the associated factors influencing the treatment course between different kinds of drugs, ages and genders; and to calculate the total cost of AChEI for whole treatment course in each patient.

\section{Subjects and methods}

\section{Diagnosis of Alzheimer's disease in mild or moderate stage}

In Taiwan, all of the hospitals have insurance contracts with the Bureau of National Health Insurance (BNHI). Therapy for dementia with AChEI is covered by insurance from BNHI but with strict payment regulations 
since 2000. Subjects using AChEI must be the patients of mild or moderate AD with complete case studies of clinical symptoms and signs, blood tests, cognitive tests and neuro-image work-ups. According to the BNHI regulations, the clinical diagnosis must conform to the criteria from the International Classification of Diseases-9 (ICD-9), or the Diagnostic and Statistical Manual of Mental Disorders-III (DSM III) or the National Institute of Neurological and Communicative Disorders and Stroke-Alzheimer's Disease and Related Disorder Association (NINDS-ADRDA), with a history of dementia for more than 6 months. Other causes of dementia must likewise be excluded. Patients with cerebral vascular disease history were excluded.

The Mini-Mental Status Examination (MMSE) score must be between 10 and 26 and clinical dementia rating (CDR) grade either 1 or 2 . The requested blood tests include venereal disease research laboratory, thyroid function, complete blood count, fasting sugar, glutamicoxaloacetic transaminase, glutamic-pyruvic transaminase, blood urea nitrogen, creatinine, serum B12 and folic acid levels. Aside from the clinical presentation, cognition tests and blood tests, all of the patients must have neuro-image studies, with either brain CT or MRI. Patients with old vascular insults, hydrocephalus, brain tumour, or any other potential cause of dementia other than Alzheimer's noted in these neuro-images were excluded. The diagnostic work-up must be performed and confirmed by a certificated neurologist or psychiatrist.

\section{Permission of AChEl prescription}

After completeness of aforementioned work-up, the clinical neurologist or psychiatrist can apply to BNHI for the permission of AChEI prescription. The entire patient' data were reviewed by an expert committee composed of BNHI neurologists or psychiatrists. The committee reviewed the history record, laboratory data, imaging data and the cognition function record. Only those who fit the criteria could be allowed to take AChEI therapy. After treatment, the patients were regularly followed-up and the cognition tests were performed every half year. If the MMSE score worsened by more than two points or the CDR worsened by 1 grade as compared with baseline, the treatment covered by the insurance of BNHI would be terminated in the panel data.

\section{Searching the drug codes and patient data}

As for the AChEI, donepezil was launched the earliest (October 1998) in Taiwan. Rivastigmine was the second (April 2000) and galantamine was the last (January 2003). Data panel from the medication file of BNHI was used to search the drug codes, which were classified by
American Hospital Formula System. There were a total seven kinds of pharmaceutical codes involving different dosages of all the aforementioned AChEI with registered licenses from BNHI. Then from the panel data by searching the drug codes, all of the patients using the medicine were collected. In this database, we were able to get the information on the patient's age, gender, start date and end date of treatment, brand name of AChEI and its dosage, cost of medicine, and whether or not changes of AChEI of each month from 2001 to 2004.

\section{Statistical analyses}

Proportion of study subjects was calculated by five age groups. Kaplan-Meier product-limit method was used to estimate duration of drug utilization. Patients with maintaining the drug prescription were censored at the end of follow-up on 31 December 2004. The log-rank test was used to compare the rates of discontinuation of treatment between patients $<76$ and $\geq 76$ years of age, which was the median age of study population. Cox proportional-hazards regression was performed to evaluate the effect of discontinuation of AChEI treatment, adjusting for age, sex and brands of AChEI. Total cost of AChEI for each patient was also calculated. A $P$-value $<0.05$ was considered significant in univariate and multivariate analyses. Statistical analyses were performed with the SAS 9.1 software package.

\section{Results}

\section{Characteristics of study targets}

The patients who fit the inclusion criteria and got the permission from BNHI for taking AChEI were consecutively enrolled. Some discontinued the treatment and some newly enrolled in the succeeding monthly record. However, the total number increased every year. The numbers of participants were 2678, 4060, 5095 and 6529 respectively from 2001 to 2004 . There were total 9877 patients, with a female predominance $(P<0.0001)$. The proportion of female was kept around $56 \%$ every year. The mean age was $75 \pm 8.2$ years. The mean age of males was $75.7 \pm 8.3$ years, which was older than the $74.7 \pm 8.0$ years in females $(P<0.0001)$. The peak of age in patients with mild to moderate AD was between 70 and 80 years. However, as classified by five age groups, there was a female preponderance in the younger age groups but a male preponderance after the age of 70 years (Fig. 1).

The number of patients treated with the three kinds of AChEI were quite different. The difference of launch time amongst these medicines influenced the proportion 


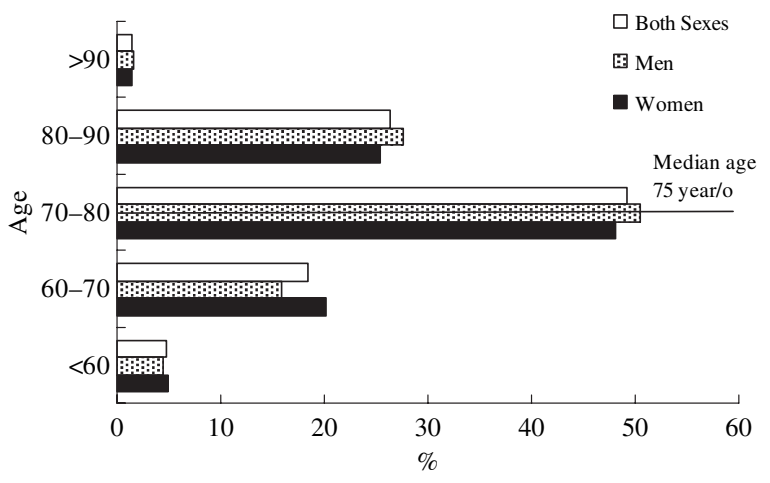

Figure 1 Proportion of target subjects by age group.

of medicine usage. Donepezil was launched early and was most commonly used, accounting for $61.3 \%$ of patients. Rivastigmine was used by $36.9 \%$ of patient. In 2003 and 2004, galantamine was started but was taken by only $1.75 \%$ of patients. There was no gender difference between male and female in choosing the AChEI $(P=0.29)$.

\section{Treatment duration and associated factors}

Even though to obtain the permission of prescription of AChEI from BNHI was not easy, around $20 \%$ of patients voluntarily dropped out of the treatment regimen before the end of 3 months. After half a year, all of the patients were asked for follow-up cognition tests, including CASI/MMSE and CDR. Around 35\% were no longer treated, partly because of the spontaneous drop-out or the termination of permission from BNHI because of the two points deterioration of MMSE or 1 grade worsening of CDR. More than half $(55 \%)$ were
Table 1 Number and percentile of patients with different durations of AChEI therapy $(n=9877)$

\begin{tabular}{lll}
\hline Total treatment duration & Patients no. & Percentile $(\%)$ \\
\hline$\leq 3$ months & 2012 & 20.37 \\
$>3$ months, $<1 / 2$ years & 1486 & 15.05 \\
$>1 / 2$ years, $<1$ year & 2020 & 20.45 \\
$1-2$ years & 2183 & 22.1 \\
$2-3$ years & 1228 & 12.43 \\
$>3$ years & 948 & 9.6 \\
\hline
\end{tabular}

not treated 1 year later and only $9.6 \%$ were treated for more than 3 years, with continued drug refill and maintaining cognition and daily function (Table 1). The mean duration of treatment was 432 days. The daily cost of AChEI therapy for AD in Taiwan was around 110 Taiwan dollars (TWD) (equivalent to $€ 2.3$ ). The average total cost of AChEI for each patient was 47593 TWD (equivalent to $€ 992$ based on the exchange rate on 21 November 2007) from the start to the termination of treatment.

As for the associated factors influencing treatment duration, we analysed the factors of different AChEI, gender, and age of 76 years old as the cut point (because 76 years was the median age of the studied population). The log-rank test showed the younger age had lower discontinuation rate of treatment $(P=0.0008)$ (Fig. 2). Proportional-hazards regression showed the old age ( $>76$ years old) was the factor associated with reduced treatment duration $(P=0.001)$. Gender $(P=0.07)$ and types of medicine $(P=0.62)$ were not significant factors. As the number of patients treated with galantamine $(n=173)$ was too small, we repeated the analysis in the database without galantamine therapy. Age was still the only significant

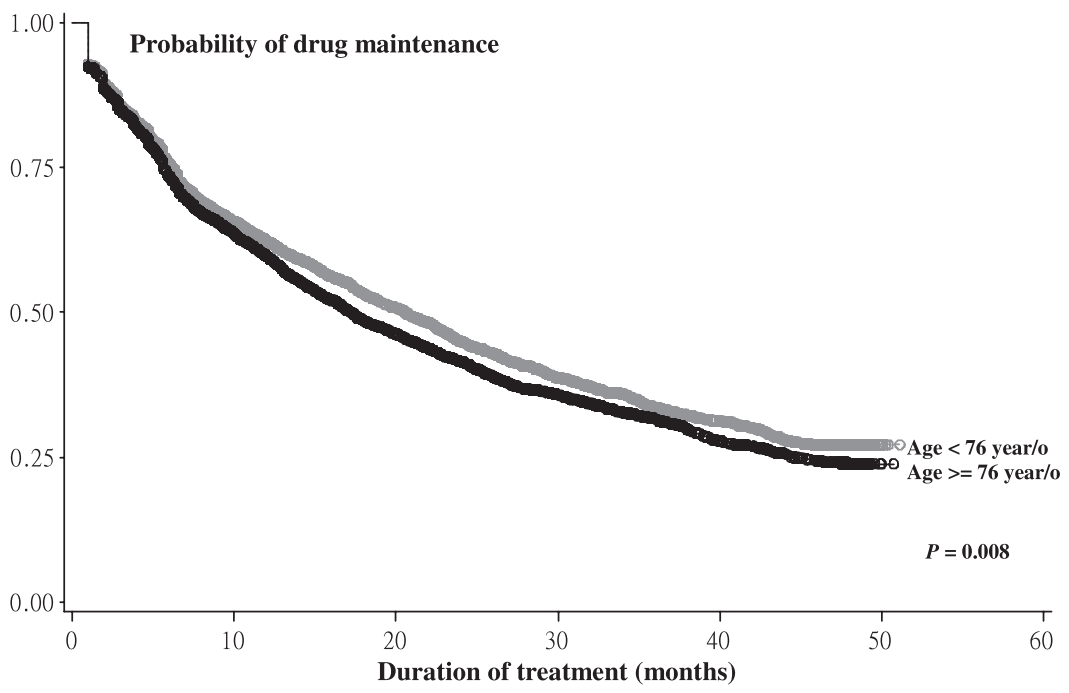

Figure 2 Kaplan-Meier curves showing the probability of drug adherence over 50 months of follow-up in patients with mild or moderate Alzheimer's dementia under AChEI therapy in 2001-2004, stratified by the age of the patients. 
associated factor of treatment duration $(P=0.0006)$ as compared with gender and drug.

\section{Discussion}

This study is the first population-based study on the long-term follow-up of AChEI therapy in patients with mild or moderate $\mathrm{AD}$, as the maintaining of cognition and daily function taken into account. Instead of searching the ICD codes from registry data in other population-based study, the diagnosis of present target subjects is made by using strict criteria as well as experts' committee review and is highly precise. It is also the first study with large database to explore the proportion of age and sex in patients with mild and moderate $\mathrm{AD}$, who were treated with the AChEI therapy. Moreover, all of the patients in this study were requested to be followed-up regularly and had cognition tests every half a year, which enabled an early detection of the discontinuation rate and the duration of maintaining both treatment and cognition. This study provides a long-term follow-up data of patients receiving treatment with AChEI.

This study showed the proportion of ages of mild to moderate $\mathrm{AD}$ was highest (49\%) at age of 70-80 years. The peak of age was younger than those reported in other epidemiological studies [11-15]. It may be because of the exclusion of patients in severe dementia in this study. As for the gender preponderance, Alzheimer's disease is more common in women than in men. The proportion of female was around $56 \%$ in this study. It was lower than those in other studies with percentage of women ranges from approximately $60 \%$ to more than $70 \%$ amongst western countries [15-17]. This study also showed the mean age of females was younger than that of males. Furthermore, as classified by five age groups, There was a female predominance in the younger age groups but after the age of 70 years, the proportion of males becomes higher. This difference can be partly attributed to lower mortality rates in women at earlier ages compared with men [14]. The patient's age shown in this panel data was the age that started AChEI treatment. This was not the onset age but reflected the age when alterations or deterioration in the patient's cognition and behaviour became noticeable, prompting family members to take the patient to the hospital for help. This study results also suggested that the age when symptoms become manifest is earlier in females than males.

This nationwide study showed that, in actual clinical practice, the rate of discontinuation is quite high amongst such population. The rate of spontaneous withdraws or discontinuation within 3 months of treatment was as high as $20 \%$. Half-a-year later, an- other $15 \%$ dropped out. Some of these patients may withdraw because of the discontinuation of insurance payments from BNHI as obvious progression of dementia was noted by cognition test after half-a-year of follow-up. After 1 year of treatment, the percentile of patients maintaining the therapy was $<45 \%$. Only $9.6 \%$ of patients were treated for more than 3 years with continued drug refill and maintenance of cognition and daily function. The mean duration in all patients was around 14 months.

Reviewing the clinical trials of $\mathrm{AD}$, the rates of adverse events (AE) were around 6-16\%, with higher rate of $\mathrm{AE}$ in the high dose of AChEI treatment [6, 18-22]. The rates of discontinuation because of $\mathrm{AE}$ were around $6-9 \%[6,18-23]$. The discontinuation rates for any reason were from $19 \%$ to $33 \%$ in these clinical trials with study period from 3 months to 1 year $[6,18$ 23]. Some of the patients may drop-out because of the side effects of medicine, whilst some may be because of comorbidities or mortality during treatment. It is indeed obvious that Alzheimer's disease considerably shortens life compared with non-Alzheimer's disease [24]. The mortality rates over 3 years in patients with dementia were from $30 \%$ to $53 \%[6,25]$. This might be one of the major causes for the low proportion of patients who can keep receiving the treatment over 3 years in our study. All the patients were old and had cognitive or behaviour disorder. This chronic disease statement may be complicated because the patients with dementia refuse (or forget) to take medicine or go to hospital, which results in high morbidity or mortality [25]. Besides, some patients appear moderate dementia instead of mild stage at first interview may be the reason why some of our study subjects discontinued their therapy as a result of obvious retrogression of cognitive function within short time-period [26,27].

In view of the published literatures on pharmacoeconomic studies $[2,3,7,9,10,28]$, the data of discontinuation rate of AChEI therapy on the Alzheimer's disease are based on the randomized controlled trial (RCT) [6, 18-23]. In the study of Neumann et al. [9], the assumed discontinuation rate is $4 \%$ in each 6 -week cycle (roughly $8 \%$ per 3 months) and $0-8 \%$ in the sensitivity analysis, which are lower than our 3 months spontaneous drop-out rate $(20 \%)$. It suggested that, there are higher adherence and lower discontinuation in experimental designed RCT than in population-based data. It may be owing to selection of the patients and the much attention the study patients receive in RCT [29]. Furthermore, most of the pharmacoeconomic studies used to simulate long-duration cohort for evaluating the cost-effectiveness of AChEI [6-10, 30-37]. The model periods were from 0.5 to 10 years $[3,4,7]$. Most of aforementioned studies did not have data of 
long-duration clinical trials and the data of long-term follow-up to estimate precisely about the rates they used for the discontinuation of treatment [6-8,30-37]. The lack of long-term follow-up data constitutes the largest uncertainty in the estimation of cost-effectiveness, and such uncertainty is introduced through modelling assumption. This present study provided the long-term data in patients with mild to moderate $\mathrm{AD}$ on keeping AChEI therapy. There showed low proportion of patients with continued treatment for more than 3 years. It is because of the common discontinuation of drug refill amongst these ageing populations and our strict regulations of treatment for only well responders.

Our study showed the discontinuation of treatment is not related to gender or the types of the AChEI. Old age was the significant risk factors for discontinuation the therapy. It may be because of the high prevalence of comorbidities, mortality and more severe demented cognition in elderly as age increased [24,25]. Under our national insurance policy with limited health budget, AChEI were only allowed to treat those good responders, who could maintain the cognition before their severe stage. With such policy and high discontinuation rate of treatment, the AChEI pharmacological cost for each patient during the whole treatment course was around 47593 TWD (equivalent to €992).

A limitation of this study is that the real and detailed causes of drop-out or discontinuation from the AChEI therapy could not be ascertained. We could not know the causes and rates of co-morbidities and mortalities amongst our targets in this database. Because we are using panel data, we could not measure the drug adherence and we could not know if the patients really took medicine or not even though they had drug refill every month. Moreover, we could not clarify the exact score of the mental function from the database. All of the patients had MMSE scores of 10-26 and CDR scores of 1-2. However, a wide variety of cognitive function, from mild to moderate demented state, is included. The drop-out rate might be varied based on the different severity of mentality function but could not be analysed in this study.

In conclusion, female were more and younger than male in mild to moderate AD. Because of the common discontinuation of drug refill amongst these ageing populations and the strict regulations of treatment for only well responders, the proportion of patients keeping AChEI therapy is low. Before disease progression to severe stage, only $9.6 \%$ of patients were treated for more than 3 years with continued drug refill and maintenance of cognition and daily function. The average duration for AChEI therapy was around 14 months. The elderly was at high risk for treatment discontinuation.

\section{References}

1. Jonsson L, Eriksdotter Jonhagen M, Kilander L, et al. Determinants of costs of care for patients with Alzheimer's disease. International Journal of Geriatric Psychiatry 2006; 21: 449-459.

2. Wimo A, Winblad B. Economic aspects on drug therapy of dementia. Current Pharmaceutical Design 2004; 10: 295-301.

3. Jonsson L. Pharmacoeconomics of cholinesterase inhibitors in the treatment of Alzheimer's disease. Pharmacoeconomics 2003; 21: 1025-1037.

4. Clegg A, Scott DA, Sidhu M, Hewitson P, Waugh N. A rapid and systematic review of the clinical effectiveness and cost-effectiveness of paclitaxel, docetaxel, gemcitabine and vinorelbine in non-small-cell lung cancer. Health Technology Assessment 2001; 5: 1-195.

5. Kaduszkiewicz H, Zimmermann T, Beck-Bornholdt HP, van den Bussche $\mathrm{H}$. Cholinesterase inhibitors for patients with Alzheimer's disease: systematic review of randomised clinical trials. British Medical Journal 2005; 331: 321-327.

6. Stewart A, Phillips R, Dempsey G. Pharmacotherapy for people with Alzheimer's disease: a Markov-cycle evaluation of five years' therapy using donepezil. International Journal of Geriatric Psychiatry 1998; 13: 445-453.

7. Wimo A. Cost effectiveness of cholinesterase inhibitors in the treatment of Alzheimer's disease: a review with methodological considerations. Drugs and Aging 2004; 21: 279-295.

8. O'Brien BJ, Goeree R, Hux M, et al. Economic evaluation of donepezil for the treatment of Alzheimer's disease in Canada. Journal of the American Geriatrics Society 1999; 47: $570-578$.

9. Neumann PJ, Hermann RC, Kuntz KM, et al. Costeffectiveness of donepezil in the treatment of mild or moderate Alzheimer's disease. Neurology 1999; 52: 11381145.

10. Migliaccio-Walle K, Getsios D, Caro JJ, Ishak KJ, O'Brien JA, Papadopoulos G. Economic evaluation of galantamine in the treatment of mild to moderate Alzheimer's disease in the United States. Clinical Therapeutics 2003; 25: 1806-1825.

11. Ferri CP, Prince M, Brayne C, et al. Global prevalence of dementia: a Delphi consensus study. Lancet 2005; 366: 2112-2117.

12. Lee C, Chang S, Su C. Neuroepidemiological study in Ilan, Taiwan (NESIT): an epidemiological survey of dementia in a rural area. Acta Neurologica Taiwanica 1997; 6: 27-35.

13. Liu HC, Lin KN, Teng EL, et al. Prevalence and subtypes of dementia in Taiwan: a community survey of 5297 individuals. Journal of the American Geriatrics Society 1995; 43: 144-149.

14. Brookmeyer R, Gray S, Kawas C. Projections of Alzheimer's disease in the United States and the public health impact of delaying disease onset. American Journal of Public Health 1998; 88: 1337-1342.

15. Rice DP, Fillit HM, Max W, Knopman DS, Lloyd JR, Duttagupta S. Prevalence, costs, and treatment of Alzheimer's disease and related dementia: a managed care perspective. The American Journal of Managed Care 2001; 7: 809-818.

16. Menzin J, Lang K, Friedman M, Neumann P, Cummings JL. The economic cost of Alzheimer's disease and related dementias to the California Medicaid program ('Medi- 
Cal') in 1995. The American Journal of Geriatric Psychiatry 1999; 7: 300-308.

17. Weiner M, Powe NR, Weller WE, Shaffer TJ, Anderson GF. Alzheimer's disease under managed care: implications from Medicare utilization and expenditure patterns. Journal of the American Geriatrics Society 1998; 46: 762770.

18. Rogers SL, Farlow MR, Doody RS, Mohs R, Friedhoff LT. A 24-week, double-blind, placebo-controlled trial of donepezil in patients with Alzheimer's disease. Donepezil Study Group. Neurology 1998; 50: 136-145.

19. Rosler M, Anand R, Cicin-Sain A, et al. Efficacy and safety of rivastigmine in patients with Alzheimer's disease: international randomised controlled trial. British Medical Journal 1999; 318: 633-638.

20. Farlow M, Anand R, Messina J Jr, Hartman R, Veach J. A 52-week study of the efficacy of rivastigmine in patients with mild to moderately severe Alzheimer's disease. European Neurology 2000; 44: 236-241.

21. Tariot PN, Solomon PR, Morris JC, Kershaw P, Lilienfeld S, Ding C. A 5-month, randomized, placebo-controlled trial of galantamine in AD. The Galantamine USA-10 Study Group. Neurology 2000; 54: 2269-2276.

22. Brodaty H, Corey-Bloom J, Potocnik FC, Truyen L, Gold M, Damaraju CR. Galantamine prolonged-release formulation in the treatment of mild to moderate Alzheimer's disease. Dementia and Geriatric Cognitive Disorders 2005; 20: $120-132$.

23. Winblad B, Engedal K, Soininen H. A 1-year, randomized, placebo-controlled study of donepezil in patients with mild to moderate AD. Neurology 2001; 57: 489-495.

24. Aguero-Torres H, Fratiglioni L, Winblad B. Natural history of Alzheimer's disease and other dementias: review of the literature in the light of the findings from the $\mathrm{Ku}$ ngsholmen Project. International Journal of Geriatric Psychiatry 1998; 13: 755-766.

25. Martin DC, Miller JK, Kapoor W, Arena VC, Boller F. A controlled study of survival with dementia. Archives of Neurology 1987; 44: 1122-1126.

26. Clarke M, Jagger C, Anderson J, Battcock T, Kelly F, Stern MC. The prevalence of dementia in a total population: a comparison of two screening instruments. Age and Ageing 1991; 20: 396-403.

27. Holmes C, Lovestone S. Long-term cognitive and functional decline in late onset Alzheimer's disease: therapeutic implications. Age and Ageing 2003; 32: 200-204.

28. Foster RH, Plosker GL. Donepezil. Pharmacoeconomic implications of therapy. Pharmacoeconomics 1999; 16: 99114.

29. Osterberg L, Blaschke T. Adherence to medication. New England Journal of Medicine 2005; 353: 487-497.

30. Caro JJ, Getsios D, Migliaccio-Walle K, Raggio G, Ward A. Assessment of health economics in Alzheimer's disease (AHEAD) based on need for full-time care. Neurology 2001; 57: 964-971.

31. Fenn P, Gray A. Estimating long-term cost savings from treatment of Alzheimer's disease. A modelling approach. Pharmacoeconomics 1999; 16: 165-174.

32. Ward A, Caro JJ, Getsios D, Ishak K, O'Brien J, Bullock R. Assessment of health economics in Alzheimer's disease (AHEAD): treatment with galantamine in the UK. International Journal of Geriatric Psychiatry 2003; 18: 740-747.

33. Garfield FB, Getsios D, Caro JJ, Wimo A, Winblad B. Assessment of Health Economics in Alzheimer's Disease (AHEAD): treatment with galantamine in Sweden. Pharmacoeconomics 2002; 20: 629-637.

34. Getsios D, Caro JJ, Caro G, Ishak K. Assessment of health economics in Alzheimer's disease (AHEAD): galantamine treatment in Canada. Neurology 2001; 57: 972 978.

35. Jonsson L, Lindgren P, Wimo A, Jonsson B, Winblad B. The cost-effectiveness of donepezil therapy in Swedish patients with Alzheimer's disease: a Markov model. Clinical Therapeutics 1999; 21: 1230-1240.

36. Hauber AB, Gnanasakthy A, Mauskopf JA. Savings in the cost of caring for patients with Alzheimer's disease in Canada: an analysis of treatment with rivastigmine. Clinical Therapeutics 2000; 22: 439-451.

37. Caro JJ, Salas M, Ward A, Getsios D, Mehnert A. Economic analysis of galantamine, a cholinesterase inhibitor, in the treatment of patients with mild to moderate Alzheimer's disease in the Netherlands. Dementia and Geriatric Cognitive Disorders 2002; 14: 84-89. 